\title{
CONF-960543--37 SANO96-0477C \\ Fielding and Calibration Issues for Diamond Photoconducting Detectors
}

Rick B. Spielman, Lawrence E. Ruggles, Richard E. Pepping,

Steven F. Breeze, John S. McGurn, and Kenneth W. Struve*

Sandia National Laboratories, Albuquerque, NM 87185-1194

Diamond photoconducting detectors are routinely fielded as soft $\mathrm{x}$-ray diagnostics on Sandia's Saturn facility. We have developed an improved detector mount that provides a 200 -ps time response, is easily cleanable, and is very rugged. In addition, we have developed a new, fast insertion unit to apply bias voltage to the detectors. Absolute calibration of the PCDs is carried out either at the Brookhaven National Synchrotron Light Source or on Sandia's laser calibration facility. We are now fielding diamond elements that have the dimensions $1 \times 3 \times 0.5 \mathrm{~mm}$ and $1 \times 1 \times 0.5 \mathrm{~mm}$. We are neutron damaging some of the $1 \times 1 \times 0.5-\mathrm{mm}$ detectors to reduce their sensitivity. We can tailor PCD sensitivity by adjusting element size and neutron damage level.

\section{INTRODUCTION}

Diamond photoconducting detectors (PCDs) $)^{1,2}$ have a number of advantages when compared to XRDs and P-I-N diodes; a rugged, readily cleanable surface, excellent time response due to the short electron/hole recombination time, low leakage current, and a low$\mathrm{Z}$ composition which minimizes the sensitivity of the detectors to higher energy $\mathrm{x}$ rays.

We describe a new PCD detector housing used to diagnose the $\mathrm{x}$-ray output from $\mathrm{z}$ pinches on Sandia National Laboratories' Saturn accelerator. ${ }^{3,4}$ The detectors are biased with custom high-bandwidth insertion units. We have been able to vary the fundamental sensitivity of diamond PCD elements by neutron damaging the diamond element in a nuclear reactor.

\section{PHOTOCONDUCTING DETECTORS}

Diamond photoconducting detectors provide an electrical output that is linearly dependent on the incident $\mathrm{x}$-ray flux and the applied bias voltage. PCDs are potentially "flat 


\section{DISCLAIMER}

This report was prepared as an account of work sponsored by an agency of the United States Government. Neither the United States Government nor any agency thereof, nor any of their employees, makes any warranty, express or implied, or assumes any legal liability or responsibility for the accuracy, completeness, or usefulness of any information, apparatus, product, or process disclosed, or represents that its use would not infringe privately owned rights. Reference herein to any specific commercial product, process, or service by trade name, trademark, manufacturer, or otherwise does not necessarily constitute or imply its endorsement, recom: mendation, or favoring by the United States Government or any agency thereof. The views and opinions of authors expressed herein do not necessarily state or reflect those of the United States Government or any agency thereof. 


\section{DISCLAIMER}

Portions of this document may be illegible in electronic image products. Images are produced from the best available original document. 
response" detectors like P-I-N diodes but without a silicon dead layer and at a much lower absolute sensitivity. The very rapid electron/hole recombination time in diamond (measured to be $\sim 200 \mathrm{ps}$ ) gives this detector excellent time response. The recombination time also directly affects the sensitivity of the detector. The shorter the recombination time the lower the detector response.

We can further reduce the intrinsic sensitivity of the detectors by placing the diamond elements in a nuclear reactor and exposing them to high levels of epi-thermal neutrons. The neutron bombardment causes lattice defects that act as recombination sites for the radiationinduced carriers. We have been able to reduce the sensitivity of the PCDs by as much as an order of magnitude with an epi-thermal neutron dose of $10^{17}$ neutrons- $\mathrm{cm}^{-2}$.

\section{DETECTOR MOUNTING}

We have designed a new, compact PCD mount based on a standard SMA connector. The standard connector is gold coated and uses a Teflon ${ }^{\mathrm{TM}}$ dielectric. The diamond element is mounted on a $1-\mathrm{cm}$-long circuit board that is designed as a $50-\Omega$ stripline. The circuit board substrate material is ceramic-loaded Teflon ${ }^{\mathrm{TM}}$. The diamond is attached to the circuit board conductors using a low temperature, indium-lead solder. (See Fig. 1.) The only significant impedance mismatches are at the coaxial-to-stripline connection and at the PCD element. The electrical time response of the mount is better than $200 \mathrm{ps}$.

The new mounting technique lets us clean the detectors in a thorough fashion. It is usual to place mounted PCDs into a ethanol-solvent ultrasound bath and clean them for several hours. After cleaning, the PCDs are first air dried and then biased at voltages reaching $500 \mathrm{~V}$ until the measured leakage current falls below several $\mathrm{pA}$. We found that this conditioning process is required for all new elements. During routine use PCDs can be cleaned as needed.

The PCDs were fielded on Saturn in a new housing capable of holding six detectors. (See Fig. 2.) The stainless-steel, vacuum-tight housing uses "SMA"-type vacuum feedthroughs for electrical connection. The detectors are located behind apertures, sweeper magnets, and x-ray filters. The magnets, providing a measured, minimum magnetic field of $1 \mathrm{kG}$, are required to remove any fast electrons produced by the z-pinch source. The design of the housing permits the application of voltages up to $1000 \mathrm{~V}$ on the PCDs. 


\section{DETECTOR BIAS}

We field these detectors in the single-ended mode to reduce electrical noise. ${ }^{2}$ This fielding technique requires high-speed, capacitive DC blocks and introduces an RC droop in the signals. Insertion units must have a high bandwidth and have a stable capacitance.

Existing commercial bias units were found to be unacceptable. One common bias unit, manufactured by Picosecond Pulse Laboratories, is the \#5532A. The 5532A has a base capacitance of $12 \mathrm{nF}$ giving a time constant of $1.2 \mu$ s when fielded in a $50-\Omega$ system. However, the bias unit has a voltage-dependent capacitance that is measured to change from $12 \mathrm{nF}$ to $5.1 \mathrm{nF}$ at an applied voltage of $1000 \mathrm{~V}$. This gives us a time constant as short as $0.51 \mu \mathrm{s}$ when the bias unit is actually operated as designed. We felt that this behavior was unacceptable.

We developed a new capacitive insertion unit based on new chip capacitor technology. Capacitors made by Wright Capacitors (3000 S. Orange Ave., Santa Anna, CA 92707, 714.546-2490) with a part number of WC2725R183KD102 had superior performance. These capacitors had a measured capacitance of $18 \mathrm{nF}$ and no measureable voltagedependent capacitance. A single capacitor is mounted in a $50-\Omega$ stripline configuration in a box using Type " $\mathrm{N}$ " connectors. A picture of the insertion unit and mounting configuration is shown in Fig. 3. The time response of two insertion units placed in series showed only a $2 \%$ difference in the input and output signal amplitudes after $600 \mathrm{ps}$ and no measureable difference between the input and output signal in after $1 \mathrm{~ns}$. The impedance of the insertion unit was measured using a Tektronix TDR Sampling Head. We measured a 100-ps mismatch of $\sim 2 \Omega$ at the capacitor and 50-ps, 4- $\Omega$ mismatches at the " $N$ " connectors.

We modeled the electrical performance of the insertion units with Sandia's SCREAMER code. ${ }^{5}$ We were able to model the RC droop of the system along with the effect of reflections. Any capacitor in the circuit creates an unavoidable impedance mismatch that causes a small signal reflection. This reflection is manifested in the measured signal by a small, apparently differentiated, signal that has the effect of cancelling the remaining signal droop. (See Figs. $4 \mathrm{a}$ and $4 \mathrm{~b}$.) Single ended detectors should be fielded with cables whose two-way length is longer than the signal plus any significant undershoot recovery. An attempt to remove the differention droop caused by the $\mathrm{RC}$ time constant will cause significant errors in the tail portion of the signal. Reflections of this nature are routinely seen on data taken at Saturn. (See Fig. 5.) 


\section{CALIBRATION}

Detector calibration is a necessary part of developing any diagnostic. We are calibrating PCDs at the National Synchrotron Light Source (NSLS) at Brookhaven National Laboratories and on a laser x-ray source at Sandia. Each method has its advantages. The synchrotron has a tuneable $\mathrm{x}$-ray beam but has a relatively non-uniform beam, a very short pulse, and is at a remote facility. The laser $\mathrm{x}$-ray source uses a 1-ns FWHM, 1.5-J laser at a wavelength of $0.5 \mu \mathrm{m}$ to vaporize and ionize the surface of targets. A careful choice of targets and filters gives us the ability to calibrate PCDs from $163 \mathrm{eV}$ to $2 \mathrm{keV}$. The laser source has a limited number of spectral points for calibration but is accurate, inexpensive, and easy to use. We use SI P-I-N diodes or X-ray CCDs as our calibrated references and flow proportional counters as an absolute standard. ${ }^{6}$

Beam non-uniformities at the NSLS make high accuracy calibrations difficult but repeated measurements have shown that the diamond PCDs have a flat spectral response over the range of $2-6 \mathrm{keV}$.

We are using our laser calibration source to get accurate calibrations at discrete $\mathrm{x}$-ray energies. For example, we use a magnesium target combined with an aluminum filter to calibrate the PCDs at $1.4 \mathrm{keV}$ or a beryllium target with a boron filter at $163 \mathrm{eV}$. Typical calibrations are in the range of $5-7 \times 10^{-4} \mathrm{~A} / \mathrm{W}-100 \mathrm{~V}$ bias depending on the individual detector. These calibrations are consistent with earlier, less accurate NSLS calibrations, calibrations by EG\&G Los Vegas (now Bechtel), and cross calibrations with other calibrated detectors such as bolometers. Fig. 6 shows the calibration of Diamond element \#25 using the NSLS at 2-6 keV and the laser plasma source at $163 \mathrm{eV}$ scaled to unity compared with a theoretical diamond PCD response for a $0.5-\mathrm{mm}$ thick element. The actual value of the calibration at $163 \mathrm{eV}$ was $7.13 \times 10^{-4} \mathrm{~A} / \mathrm{W}-100 \mathrm{~V}$.

\section{Effects of Neutron Damage on Diamond}

We compared the sensitivity of neutron-damaged PCDs with non-neutron-damaged PCDs on Saturn by fielding the detectors with identical filters at identical $\mathrm{x}$-ray fluxes on the same shots. The detectors were found to perform similarily with or without neutron damage with the sole exception of sensitivity. Figure 7 shows a comparison of two PCDs, one neutron damaged at a level of $1 \times 10^{16} \mathrm{n}-\mathrm{cm}^{-2}$ of epithermal neutrons. We found that the sensitivity of the neutron-damaged PCD was $10 \mathrm{X}$ lower than the sensitivity of the undamaged PCD. 


\section{CONCLUSIONS AND FUTURE PLANS}

We routinely use diamond PCD detectors for z-pinch experiments on Saturn. These detectors are trouble free, reliable, and very easy to field. The new mount coupled with the extreme ruggedness of diamond makes cleaning a simple process. We hope to extend the useable spectral range of diamond PCDs down to $50 \mathrm{eV}$.

\section{ACKNOWLEDGEMENTS}

The development and manufacture of the diamond photoconductor elements themselves was solely due to the efforts of SUNOL Technologies, Inc. Neutron damaging to reduce diamond sensitivity was demonstrated by $\mathrm{S}$. Han at Los Alamos National Laboratories in 1994. We would like to thank the Sandia reactor group in providing the nuclear reactor and the necessary expertise in order to provide quantitative levels of neutron damage on the diamond. This work was supported by the US Department of Energy under Contract No. DE-AC04-94AL85000.

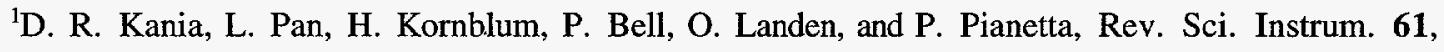
2765 (1990).

${ }^{2}$ R. B. Spielman, Rev. Sci. Instrum. 63, 5056 (1992).

${ }^{3}$ D. D. Bloomquist, R. W. Stinnett, D. H. McDaniel, J. R. Lee, A. W. Sharpe, J. A. Halbleib, L. G. Schlitt, P. W. Spence, and P. Corcoran, Proc. of the Sixth IEEE Pulsed Power Conference, Arlington, VA edited by P. J. Turchi and B. H. Bernstein (IEEE, New York, 1987), p. 310.

${ }^{4}$ R. B. Spielman. R. J. Dukart, D. L. Hanson, B. A. Hammel, W. W. Hsing, M. K. Matzen, and J. L. Porter, Proc. of the Second Int. Conference on Dense Z-Pinches, Laguna Beach, CA edited by N. R. Pereira, J. Davis, and N. Rostoker (AIP Conference Proc. 195, New York, 1989), p. 3.

${ }^{5}$ M. L. Kiefer, K. L. Fugelso, K. W. Struve, M. M. Widner, SCREAMER, A Pulsed Power Design Tool, 25 August 1995 (Sandia Internal Document).

${ }^{6}$ L. E. Rugges and J.L. Porter, "Calibration of a Time-Resolving Spectrometer in the 100 to $800 \mathrm{eV}$ Spectral Region", Proc. of 11th Topical High Temperature Plasma Diagnostics Conf., Monterey, CA May 1996. 
Figure Captions

Fig. 1. A $1 \mathrm{X} 3-\mathrm{mm}$ diamond PCD element is shown mounted on the Sandia stripline mount.

Fig. 2. The PCDs are mounted in a stainless steel housing that has integral X-ray filter holders and sweeper magnets.

Fig. 3 The Sandia-designed insertion unit is shown. The DC blocking capacitor is mounted in a $50-\Omega$ stripline configuration. A $100-\mathrm{k} \Omega$ resistor is used to place a DC bias voltage on the unit.

Fig. 4a. Apulse is generated by a variable resistance load on a biased 20 -ns long $50-\Omega$ cable and is measured as a voltage across a 50- $\Omega$ load.

Fig. 4b. The same pulse in Fig. 4A is shown with the reflection caused by the capacitive insertion unit.

Fig. 5. A typical pulse from an $\mathrm{x}$-ray detector showing the reflection caused by the insertion capacitor.

Fig. 6. Calibration data for Diamond Element \# 25 is superimposed on a theoretical diamond absorption curve (scaled to unity). The size of the boxes are indicative of the calibration errors.

Fig. 7. A neutron-damaged PCD (dashed curve) is compared with an undamaged normal diamond PCD (solid curve). 


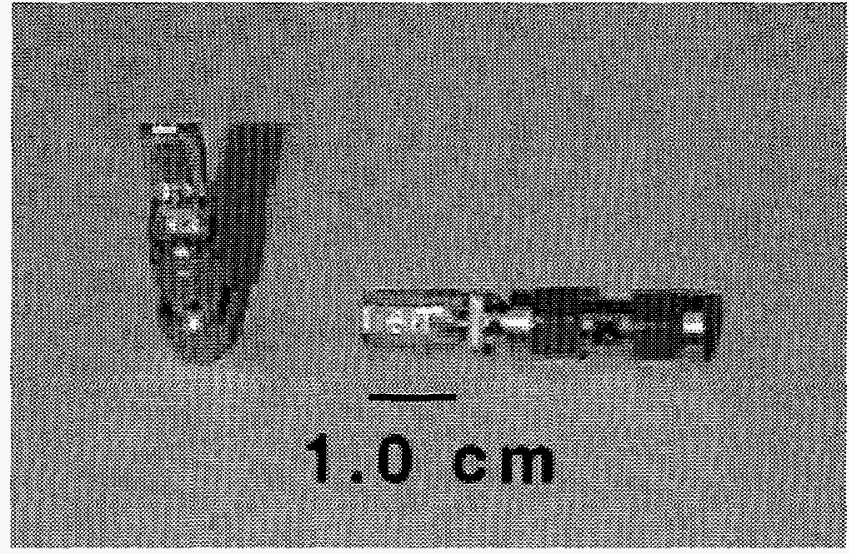

Fig. 1. A $1 X 3-m m$ diamond PCD element is shown mounted on the Sandia stripline mount. 


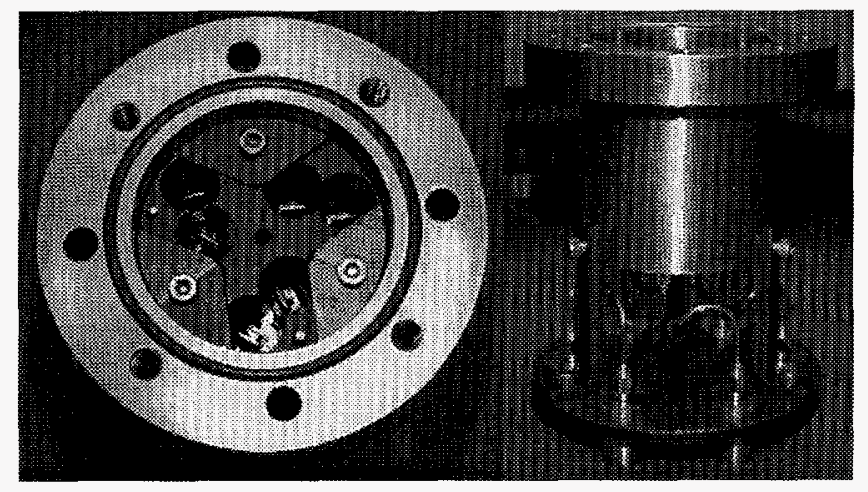

Fig. 2. The PCDs are mounted in a stainless steel housing that has integral $x$-ray filter holders and sweeper magnets. 


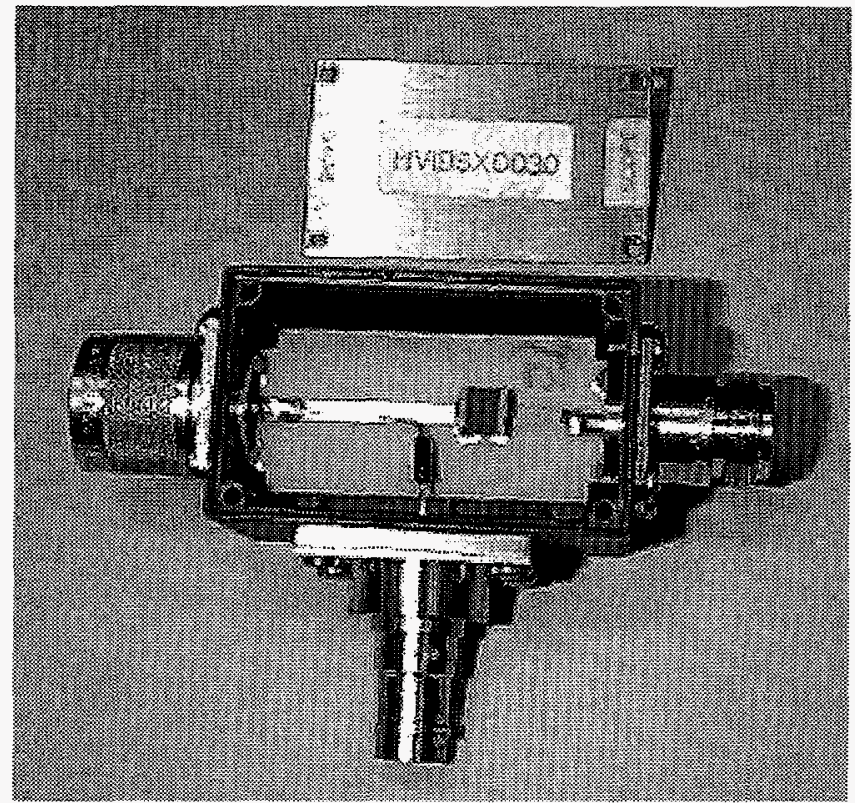

Fig. 3. The Sandia-designed insertion unit is shown. The DC blocking capacitor is mounted in a $50-\Omega$ stripline configuration. A $100-\mathrm{k} \Omega$ resistor is used to place a DC bias voltage on the unit. 


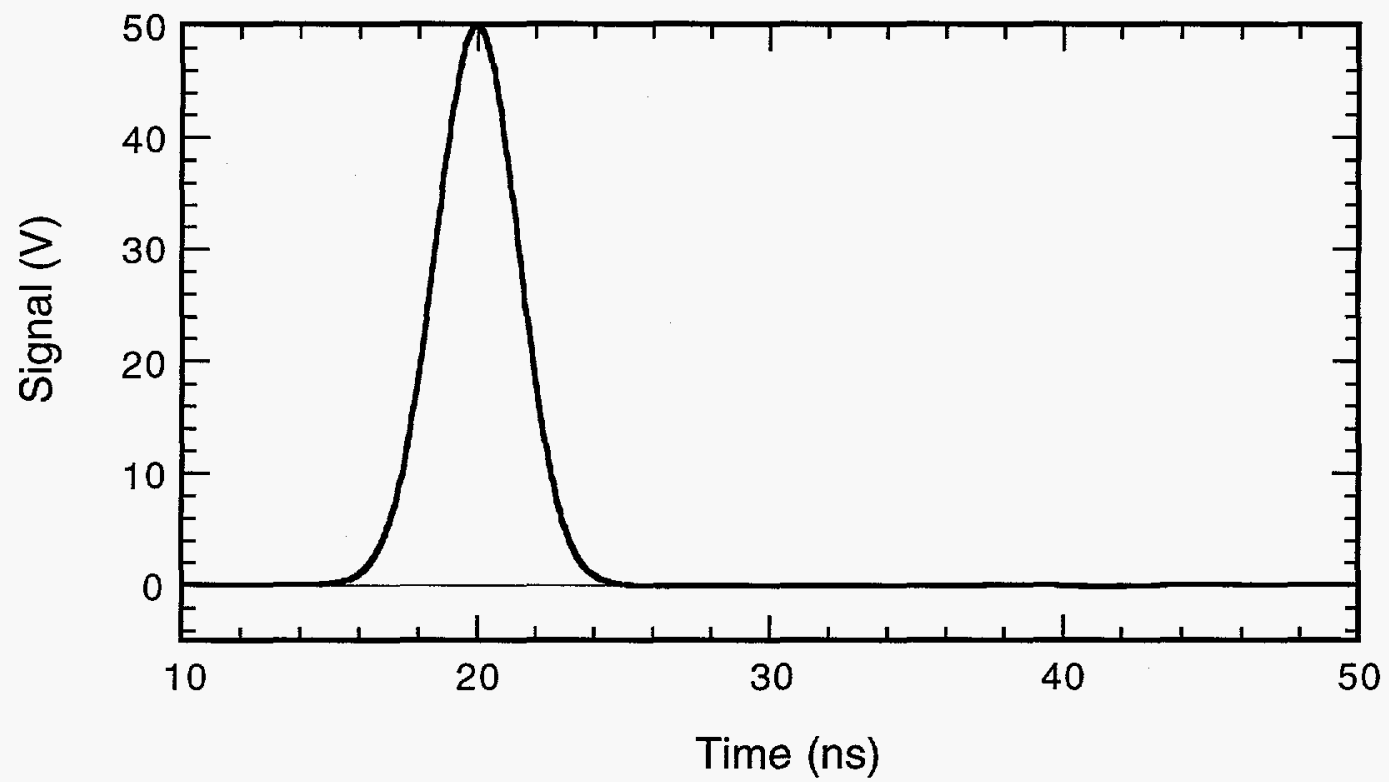

Fig. 4a. A pulse is generated by a variable resistance load on a biased 20 -ns long $50-\Omega$ cable and is measured as a voltage across a $50-\Omega$ load. 


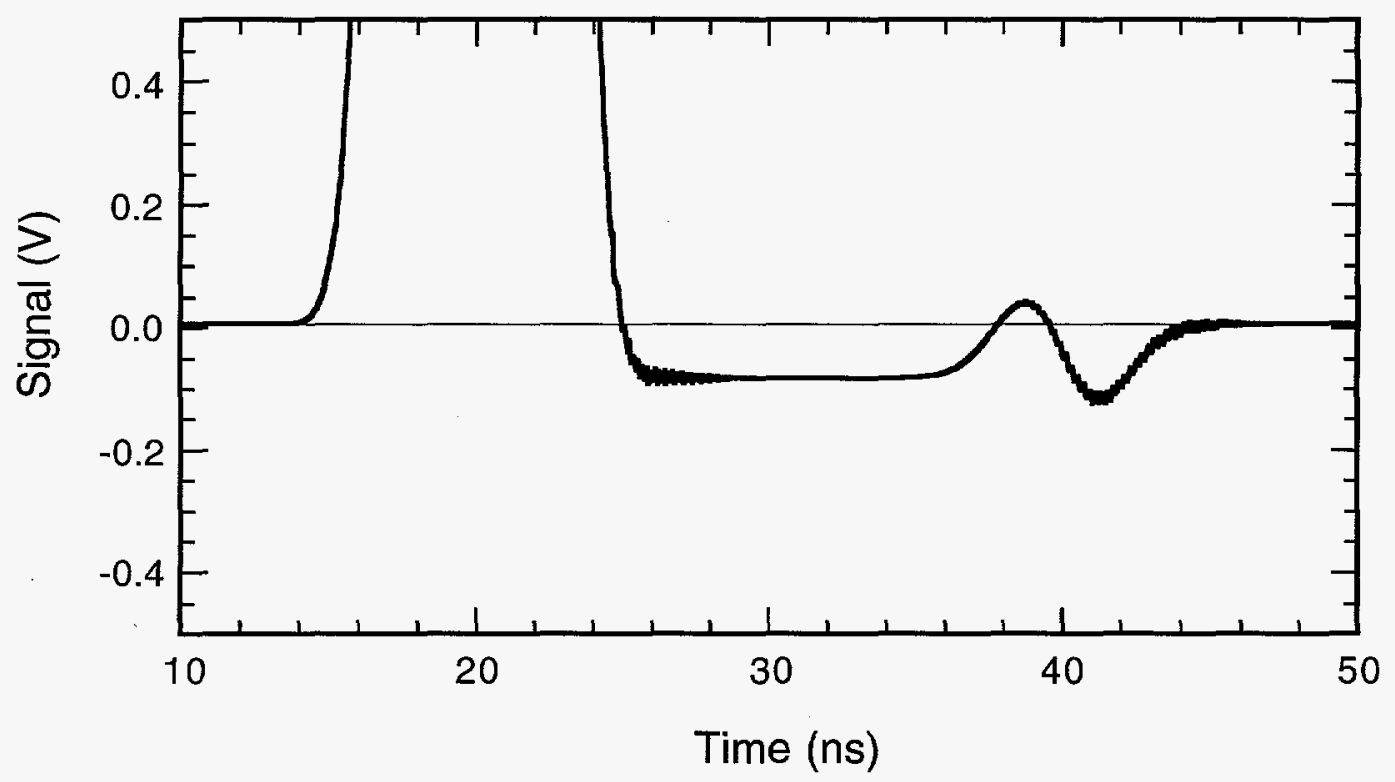

Fig. $4 \mathrm{~b}$. The same pulse in Fig. $3 \mathrm{~A}$ is shown with the reflection caused by the capacitive insertion unit. 


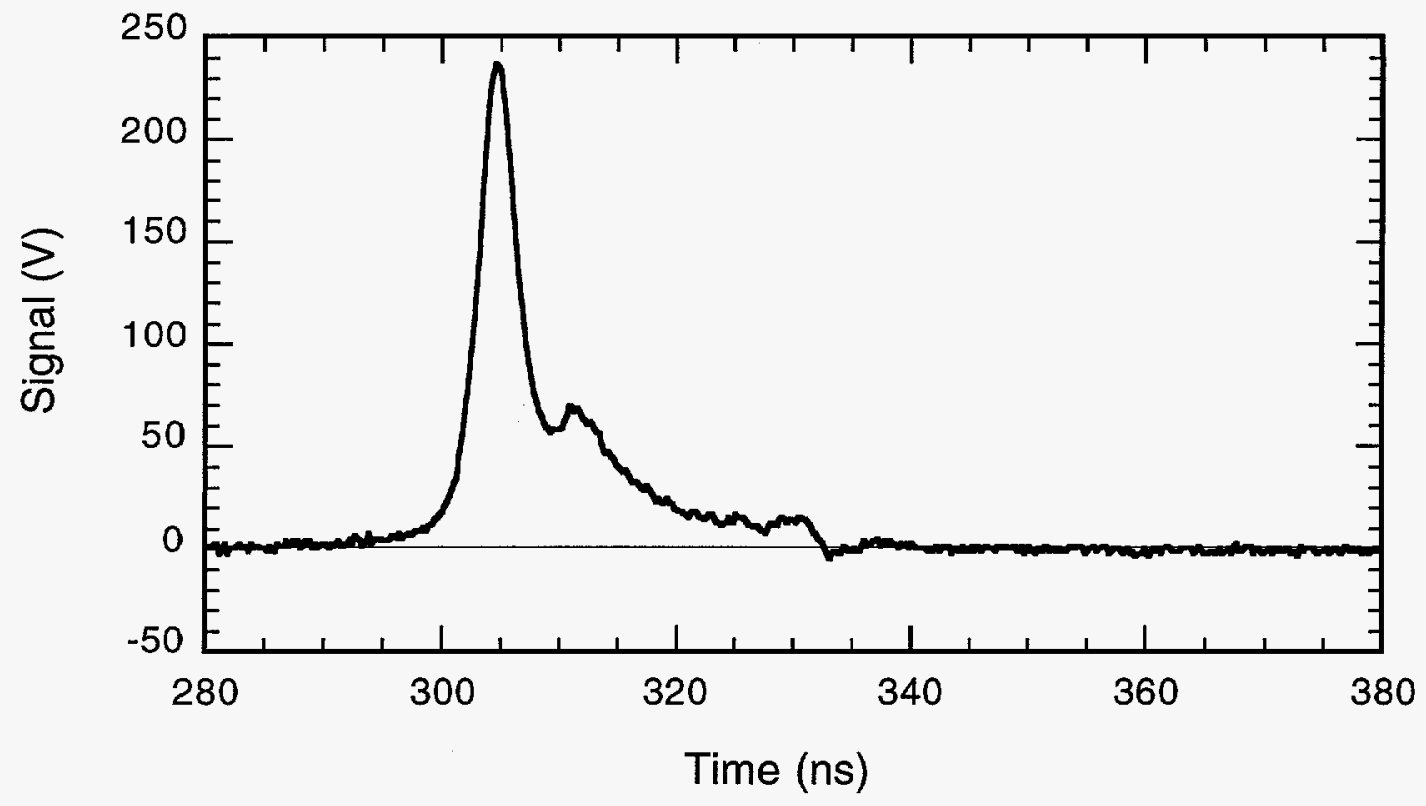

Fig. 5. A typical pulse from an x-ray detector showing the reflection caused by the insertion capacitor. 


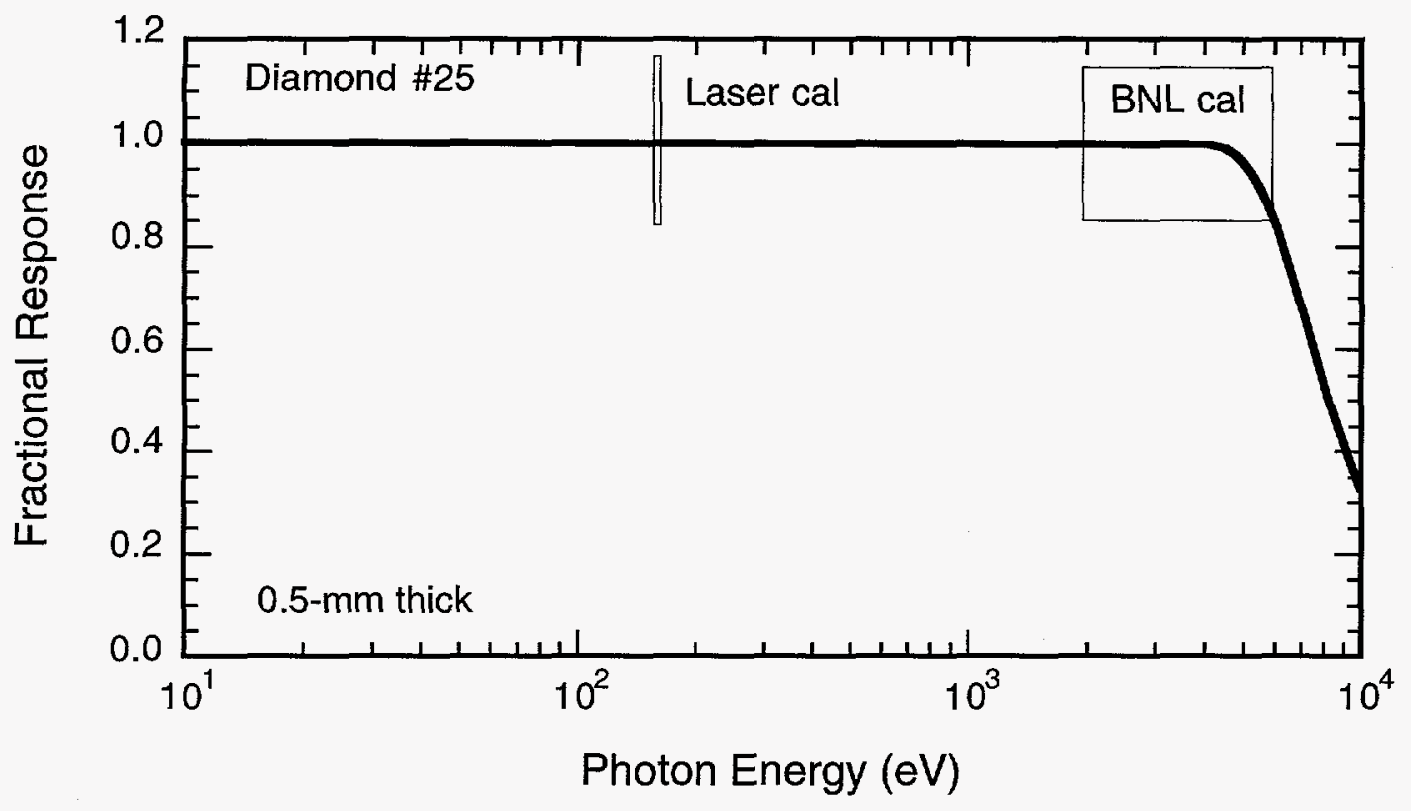

Fig. 6. Calibration data for Diamond Element \# 25 is superimposed on a theoretical diamond absorption curve (scaled to unity). The size of the boxes are indicative of the calibration errors. 


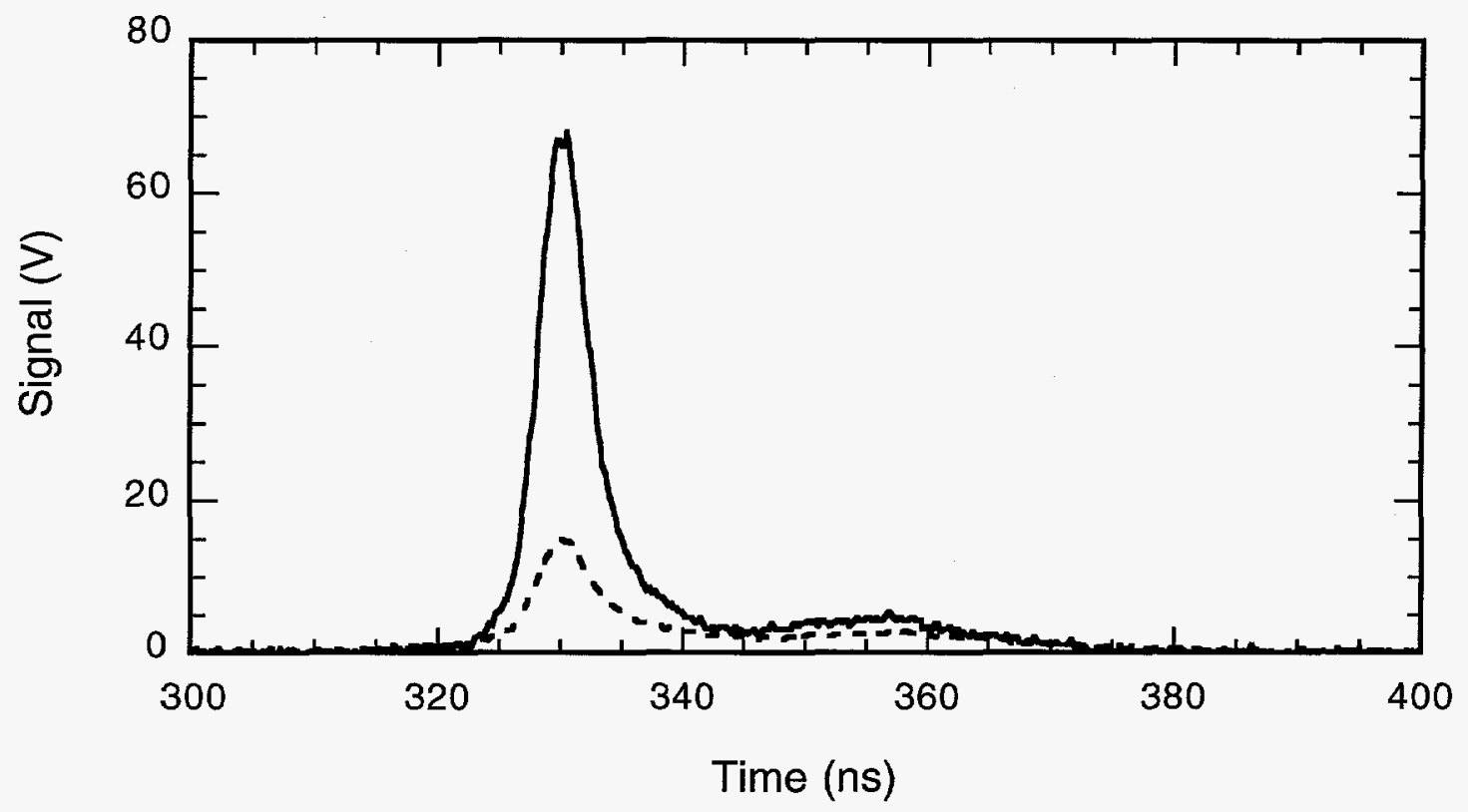

Fig. 7. A neutron-damaged PCD (dashed curve) is compared with an undamaged normal diamond PCD (solid curve). 